\title{
The Mental Health Impact of COVID-19: Salisbury District Hospital
}

\author{
Sabrina Carta, Frances Ng \\ Anaesthetics Department, Salisbury District Hospital, Salisbury, Wiltshire \\ Email: Sabrina.carta@nhs.net
}

How to cite this paper: Carta, $\mathrm{S}$. and $\mathrm{Ng}$, F. (2021). The Mental Health Impact of COVID-19: Salisbury District Hospital. Psychology, 12, 1118-1126.

https://doi.org/10.4236/psych.2021.127068

Received: June 3, 2021

Accepted: July 19, 2021

Published: July 22, 2021

Copyright $\odot 2021$ by author(s) and Scientific Research Publishing Inc. This work is licensed under the Creative Commons Attribution International License (CC BY 4.0).

http://creativecommons.org/licenses/by/4.0/

\section{(c) (i) Open Access}

\begin{abstract}
Background: With 1.5\% of hospital presentations in Salisbury district hospital in 2019 being attributed to mental health issues, this paper aims to highlight the indirect impact COVID-19 has had on mental health burden in a district general hospital, in southern England. Methods: A retrospective analysis was carried out on intensive care unit (ICU) records between March $1^{\text {st }}$ and May $31^{\text {st }} 2018$, 2019 and 2020 relating to total admissions and mental health presentations. Data relating to referrals was analysed from the mental health liaison team (MHLT) for the above monthly periods in 2019 and 2020. Last of all, information relating to emergency department (ED) total and mental health presentations were collected via the trust informatics team for the same period in 2018, 2019 and 2020. Data collected were statistically analysed using the two-proportion z-test. Results: Data from ICU showed a $15.7 \%$ increase in specified mental health presentations from 2019 to 2020, compared to a $0.65 \%$ decrease from 2018 to 2019 . The MHLT report revealed no statistical difference in the number of referrals made between 2019 and 2020. ED data showed mental health issues made up $2.06 \%$ of all presentations in 2020, a statistically significant increase compared to $1.53 \%$ in 2019 . Conclusion: Despite fewer overall admissions to ICU and presentations to ED at Salisbury District Hospital in 2020, the percentage of these due to self-harm, overdose and suicidal attempts have increased compared to the same period in 2019 and 2018. Literature remains limited but the long-term consequences on the mental health of the population are to be expected.
\end{abstract}

\section{Keywords}

COVID-19, Mental Health, Presentations

\section{Background}

Salisbury District Hospital managed by Salisbury NHS Foundation Trust has a 
470-bed capacity, 12 within the Intensive Care Unit (ICU), with 51,278 presentations to the Emergency Department (ED) in 2019.

Unsurprisingly, the editorial team of the Lancet highlights the accentuation of neglect of those with severe mental illness during the COVID-19 pandemic (Editorial Lancet Psychiatry, 2020). Clinically, the apparent increase in mental healthrelated intensive care admissions coupled with the limited literature has resulted in the need for this study. In this review we will explore a three-month period in 2018, 2019 and 2020, giving a snapshot of the burden on the National Health Service (NHS) from anxiety, depression, suicide, and harmful behaviours such as self-harm and overdose. We hope to highlight the indirect impact that COVID-19 has had on the mental health burden in a district general hospital (DGH) in southern England.

\section{Methods}

A retrospective analysis was carried out of intensive care records from between March $1^{\text {st }}$ and May $31^{\text {st }} 2018,2019$ and 2020, with this period chosen due to the period of lockdown in England, during which significant restrictions were introduced.

Data were sought from the Intensive care national audit \& research centre (ICNARC), where primary search criteria utilised was patients with a primary or secondary reason for admission of "self-harmand" "overdose". Data from ICNARC were thereafter compared with the paper-based intensive care admissions book to ensure any outliers from coding were identified.

Further data were collected from the mental health liaison team (MHLT) for the monthly periods set in both 2019 and 2020. Information was collated and cases reviewed individually against a referrals data sheet (Appendix A) to ensure only those presenting with deliberate self-harm, overdose, suicidal ideation and attempted suicide were included.

Lastly, information relating to ED presentations was sought via the informatics team within the trust for the same three-month period in 2018, 2019 and 2020. Data included the total number of presenting patients to ED along with those presenting with deliberate self-harm, suicidal intent, lacerations, anxiety, depression, psychosis and overdose. Each identified patient was searched on software e-WinDIP Live, which provides historic information on presentations and admissions, confirming the accuracy of initial coding.

Comparison of data between years was subjected to statistical analysis using the two-proportion z-test, as per the BMJ significance test for a difference in two proportions which takes into account the variation in sample sizes between years (BMJ, 2020).

\section{Results}

Data from ICU between March 2018 and May 2018 inclusive, showed six (6) admissions for level 2 or 3 care related to self-harm or overdose, representing 
4.95\% (6/121) of admissions. Between March 2019 and May 2019 inclusive, there were only five (5) such admissions for level 2 or 3 care out of 116 total admissions, indicating that those with the specified mental health issues made up $4.3 \%$ of admissions. This contrasts the same period in 2020 in which there were seventeen (17) admissions of a total 85 (20\%), a statistically significant absolute increase of $15.7 \%$ from 2019 ( $p<0.001,95 \%$ CI 6.95 - 24.4) (Table 1), which reflects a remarkable relative increase of $365 \%$ (15.7/4.3).

Data from the MHLT report show that the number of intra-hospital referrals for self-harm, overdose, suicide attempt or ideation made up 58\% (61/105), 54\% (54/100) and 61\% (75/122) of total referrals to MHLT for March, April and May 2019, respectively. The same data was collated from March, April and May 2020 which has shown 65\% (62/96), 57\% (39/69) and 58\% (46/80) respectively with statistical analysis revealing no significant difference between the two years $(p=$ $0.65,95 \%$ CI (-6.25 - 10.1)) (Table 2).

Analysing ED data, the percentage of presentations to ED following the set criteria (classed in our study as visits coded with deliberate self-harm, suicidal intent, lacerations, anxiety, depression, psychosis and/or overdose) between March and May 2018 inclusive was 1.75\% (207/11 861). In the same three-month period in 2019, presentations for mental health issues made up $1.53 \%$ of presentations (194/12 636). This is compared to the 2020 data, where $2.06 \%$ of presentations were related to mental health (186/8997), a statistically significant absolute increase from the previous year of $0.53 \%(p<0.01,95 \%$ CI $0.18-0.89)$ (Table $3)$, reflecting a relative increase of $34.6 \%(0.53 / 1.53)$.

Table 1. ICU data.

\begin{tabular}{lc}
\hline & March-May \\
\hline 2018 & $6 / 121(4.96 \%)$ \\
2019 & $5 / 116(4.31 \%)$ \\
2020 & $17 / 85(20 \%)$ \\
\hline
\end{tabular}

Table 2. MHLT data.

\begin{tabular}{ccccc}
\hline & March & April & May & Total \\
\hline 2019 & $61 / 105(58 \%)$ & $54 / 100(54 \%)$ & $75 / 122(61 \%)$ & $190 / 327(58.1 \%)$ \\
2020 & $62 / 96(65 \%)$ & $39 / 69(57 \%)$ & $46 / 80(58 \%)$ & $147 / 245(60 \%)$ \\
\hline
\end{tabular}

Table 3. ED data.

\begin{tabular}{ccccc}
\hline & March & April & May & Total \\
\hline 2018 & $75 / 3660(2.05 \%)$ & $64 / 3865(1.65 \%)$ & $68 / 4316(1.58 \%)$ & $207 / 11861(1.75 \%)$ \\
2019 & $61 / 4253(1.43 \%)$ & $64 / 4054(1.58 \%)$ & $69 / 4329(1.59 \%)$ & $194 / 12636(1.53 \%)$ \\
2020 & $78 / 3228(2.42 \%)$ & $55 / 2463(2.23 \%)$ & $53 / 3306(1.60 \%)$ & $186 / 8997(2.06 \%)$ \\
\hline
\end{tabular}




\section{Discussion}

\subsection{A Greater Burden of Mental Health}

The three-month period between March and May 2020, coinciding with the start of the COVID-19 pandemic in the UK, has seen an increase in the proportion of mental health presentations at Salisbury District Hospital. This is most evident in the $15.7 \%$ absolute increase (which reflects a remarkable $365 \%$ relative increase) in the percentage of intensive care admissions relating to self-harm or overdose in 2020 compared to the same period in the previous two years.

This trend is mirrored in the Emergency Department (ED), where the percentage of presentations attributable to specified mental health conditions has also significantly increased between March to May 2020 compared to the same period in 2019.

This observation is unique to 2020, as comparing 2019 with 2018 data over these same three months yields no significant difference in percentage of mental health-related presentations, in either the ICU or ED setting. This supports the theory that the COVID-19 pandemic is a major contributory factor to worsening mental health in 2020 rather than an inherent increase year-on-year.

It is likely that the government-mandated lockdown restrictions have increased the incidence of mental health problems during the pandemic. Recently published reviews and meta-analyses from China and Italy found high rates of depression, anxiety and insomnia in the general public, patients and healthcare workers, with female gender and young age being particularly high risk groups (Li et al., 2020; Rossi et al., 2020).

Although more studies on the effects of COVID-19 on community healthcare are awaited, anecdotal evidence of reduced access to community mental health services and GPs due to staff sickness, re-allocation of staff to acute care specialities, and enforced cancellation of non-urgent appointments may explain the upward trend in ED and ICU mental health presentations, as patients may bypass primary care and present later on at a more severe stage of mental illness.

There may also be a direct effect of infection with COVID-19 virus on neurological and psychiatric state. Whilst a meta-analysis of patients recovering from the similar severe acute respiratory syndrome (SARS) and middle east respiratory syndrome (MERS) viruses have suggested no neurological sequelae (Rogers et al., 2020), newer studies on COVID-19 suggest survivors are at increased risk of short- and long-term neuropsychiatric symptoms, which may result from COVID-19 induced ischaemic changes and neuronal damage evidenced at autopsy (Taquet et al., 2021; Boldrini et al., 2021). In a review by Righy et al. (2019), rates of post-traumatic stress disorder (PTSD) after a prolonged ICU stay were similar to those in civil war survivors and humanitarian disasters, and persisted even 12 months afterwards.

\subsection{Fewer Admissions and Presentations Due to Other Causes}

Interestingly while the proportion of mental health presentations increased, 
overall patient numbers admitted to ICU and presenting to ED during March to May 2020 was reduced. ICU saw a $27 \%$ drop in total admissions whilst total number of ED presentations were reduced by $29 \%$, compared to the same period in 2019. These figures reflect those of emergency departments across the United Kingdom (UK) - there were fewer road traffic accidents, other trauma, chronic disease presentations, social admissions and iatrogenic harm due to surgery or chemotherapy (Vaughan et al., 2020; NHS England, 2020a, 2020b).

This is likely a direct result of government guidelines instructing the general public to stay at home, avoid travelling, and the cancellation of elective surgical procedures and non-urgent outpatient appointments. It may also reflect the attitude of the general public in viewing hospitals as high-risk areas and not attending unless in extremis, a finding reported in one US survey (Czeisler, 2020).

\subsection{No Change in Intra-Hospital Mental Health Referrals}

The data from the mental health liaison team, in contrast to the ICU and ED data, showed no significant change in intra-hospital referrals related to self-harm, overdose or suicidal ideation between 2020 and 2019, although there have been fewer referrals in general (245 in 2020 compared to 327 in 2019). It is unclear why this is the case, and there appears to be a discrepancy between patients presenting with mental health issues and subsequent referrals to the MHLT.

\subsection{Future Outlook}

The ramifications of COVID-19 on the mental health of patients and healthcare staff are significant and likely to continue for some time. A study of Chinese health workers by Wu et al. (2009) showed that $40 \%$ of staff continued to display signs and symptoms of PTSD even three years on from the SARS epidemic.

The unprecedented nature of government involvement and nationwide lockdown has had a huge economic impact, disrupting employment and education as well as the public outlook on healthcare and social interaction. The long-term psychological effect, particularly on children and adolescents is yet to be established.

In a more positive vein, there are a growing number of technological advances that aim to improve mental health and facilitate the provision of healthcare, and the restrictions imposed by the COVID-19 pandemic may inadvertently promote their use. Examples include phone applications for meditation and improving sleep (Bresler, 2021); online cognitive behavioural therapy (CBT); artificial intelligence that recognises people at risk of suicide and alerts local volunteers to come to their aid ( $\mathrm{Li}$ et al., 2020). Many employers have found they are able to maintain staff working from home, reducing commuting time and enabling families to spend more time together. More studies are needed to identify the vulnerable populations that would benefit most from these interventions, and to assess their effectiveness. 


\section{Conclusion}

Despite fewer overall admissions to ICU and presentations to ED at Salisbury District Hospital over March-May 2020 compared to the same three-month period in 2019 and 2018, the percentage of these due to self-harm, overdose and suicidal attempts have increased, possibly due to an unmet increased demand for community mental health services during the COVID-19 pandemic. Similar patterns are emerging in studies elsewhere in the UK and internationally, and long-term consequences on the mental health of the population are expected.

\section{Limitations}

This is a retrospective study and confounding factors cannot be excluded. The sample sizes from ICU and MHLT data are small, and despite best efforts to limit selection and classification bias by standardising specific criteria for mental health presentations across our three datasets, they are difficult to completely eliminate and are dependent on correct coding. Further studies, with larger patient groups over a greater timespan, may shed light on the long-term effects of the COVID-19 pandemic on mental health, and may allow detailed subgroup analyses to identify those at highest risk, for which intervention could be targeted.

\section{Availability of Data and Materials}

The data that support the findings of this study are available from Salisbury District Hospital informatics department but restrictions apply to the availability of these data, which were used under license for the current study, and so are not publicly available. Data is however available from the authors upon reasonable request and with permission of Salisbury District Hospital informatics department.

\section{Authors' Contributions}

SC sought, analysed and interpreted the ED, ICU and MHLT data sets and was a major contributor to the writing of the abstract, background and methodology. FN was a major contributor in writing the discussion and conclusions. All authors read and approved the final manuscript.

\section{Acknowledgements}

Dr. Ian Jenkins-Salisbury District Hospital.

\section{Conflicts of Interest}

The authors declare no conflicts of interest regarding the publication of this paper.

\section{References}

BMJ (2020). Differences between Percentages and Paired Alternatives. 
https://www.bmj.com/about-bmj/resources-readers/publications/statistics-square-one/ 6-differences-between-percentages-and

Boldrini, M., Canoll, P. D., \& Klein, R. S. (2021). How COVID-19 Affects the Brain. JAMA Psychiatry.

Bresler, T. (2021). Headspace: Staying Grounded during the Pandemic.

Czeisler, M.É., Marynak, K., Clarke, K.E.N. et al. (2020). Delay or Avoidance of Medical Care Because of COVID-19-Related Concerns-United States. Morbidity and Mortality Weekly Report, 69, 1250-1257.

Editorial Lancet Psychiatry (2020). Mental Health and COVID-19: Change the Conversation. Lancet Psych, 7, 463.

https://digital.hbs.edu/platform-digit/submission/headspace-staying-grounded-duringthe-pandemic/

Li, S., Yang, L., Zhang, C., Xiang, Y. T., Liu, Z., Hu, S., \& Zhang, B. (2020). Online Mental Health Services in China during the COVID-19 Outbreak. The Lancet Psychiatry, 7, e18.

NHS England (April 2020b). A\&E Attendances and Emergency Admissions April 2020 Statistical Commentary https://www.england.nhs.uk/statistics/wp-content/uploads/sites/2/2020/04/Statistical-c ommentary-April-2020-jf8hj.pdf

NHS England (March 2020a). A\&E Attendances and Emergency Admissions March 2020 Statistical Commentary.

https://www.england.nhs.uk/statistics/wp-content/uploads/sites/2/2020/04/Statistical-c ommentary-March-2020-jf8hj.pdf

Righy, C., Rosa, R. G., da Silva, R. T. A. et al. (2019). Prevalence of Post-Traumatic Stress Disorder Symptoms in Adult Critical Care Survivors: A Systematic Review and Meta-Analysis. Critical Care, 23, 213.

Rogers, J., Chesney, E., Oliver, D., Pollak, T., McGuire, P., Fusar-Poli, P., Zandi, M., Lewis, G., \& David, A. (2020). Psychiatric and Neuropsychiatric Presentations Associated with Severe Coronavirus Infections: A Systematic Review and Meta-Analysis with Comparison to the COVID-19 Pandemic. The Lancet Psychiatry, 7, 611-627.

Rossi, R., Socci, V., Talevi, D., Mensi, S., Niolu, C., Pancitti, F., Di Marco, A., Rossi, A., Siracusano, A., \& Di Lorenzo, G. (2020). COVID-19 Pandemic and Lockdown Measures Impact on Mental Health among the General Population in Italy, An N=18147 Web-Based Survey. MedRxiv.

Taquet, M., Luciano, S., Geddes, J. R., \& Harrison, P. J. (2021). Bidirectional Associations between COVID-19 and Psychiatric Disorder: Retrospective Cohort Studies of 62354 COVID-19 Cases in the USA. The Lancet Psychiatry, 8, 130-140.

Vaughan, L. (2020). Where Are the Patients? The Factors Affecting the Use of Emergency Care during COVID-19.

https://www.nuffieldtrust.org.uk/news-item/where-are-the-patients-the-factors-affecti ng-the-use-of-emergency-care-during-covid-19

Wu, P., Fang, Y., Guan, Z., Fan, B. et al. (2009). The Psychological Impact of the SARS Epidemic on Hospital Employees in China: Exposure, Risk Perception, and Altruistic Acceptance of Risk. The Canadian Journal of Psychiatry, 54, 302-311. 


\section{Appendix A: Mental Health Liaison Salisbury District Hospital-Referral/Data Form}

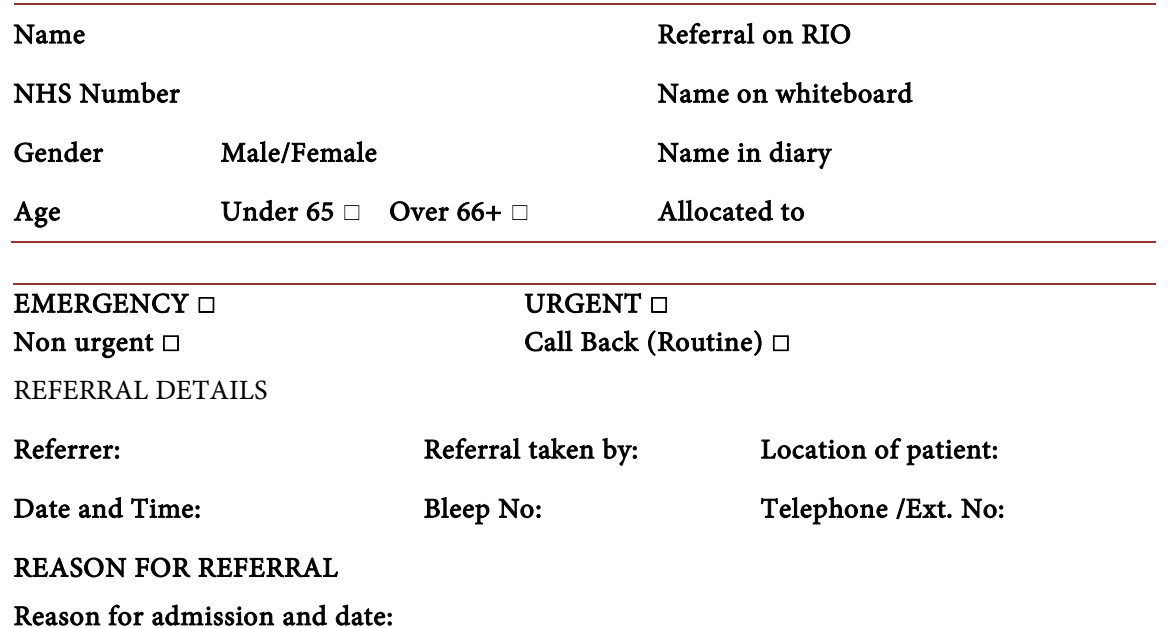

Mental health concerns:

Medical comorbidities:

Any other relevant information:

\section{RAM Score}

Family Household (under 18's only)

\begin{tabular}{lll}
\hline Name & DoB & Address if different \\
\hline
\end{tabular}

\begin{tabular}{lll}
\hline Alcohol & Illicit drugs & Self harm/OD \\
Psychosis & Depression/anxiety & Bi polar \\
dementia & Maternity & Somatoform \\
Delirium & Suicidal ideas & Personality disorder \\
Behaviour & Medication & Clozapine \\
Other (please specify) & & \\
From AWP Bed & & Wilts/Dorset/Somerset/Hamp/ \\
Assessed & CCG Area (GP location) & Other/NFA \\
Reason not assessed & Yes/No & Call back-Seen/declined/Not seen Inappropriate Ref
\end{tabular}




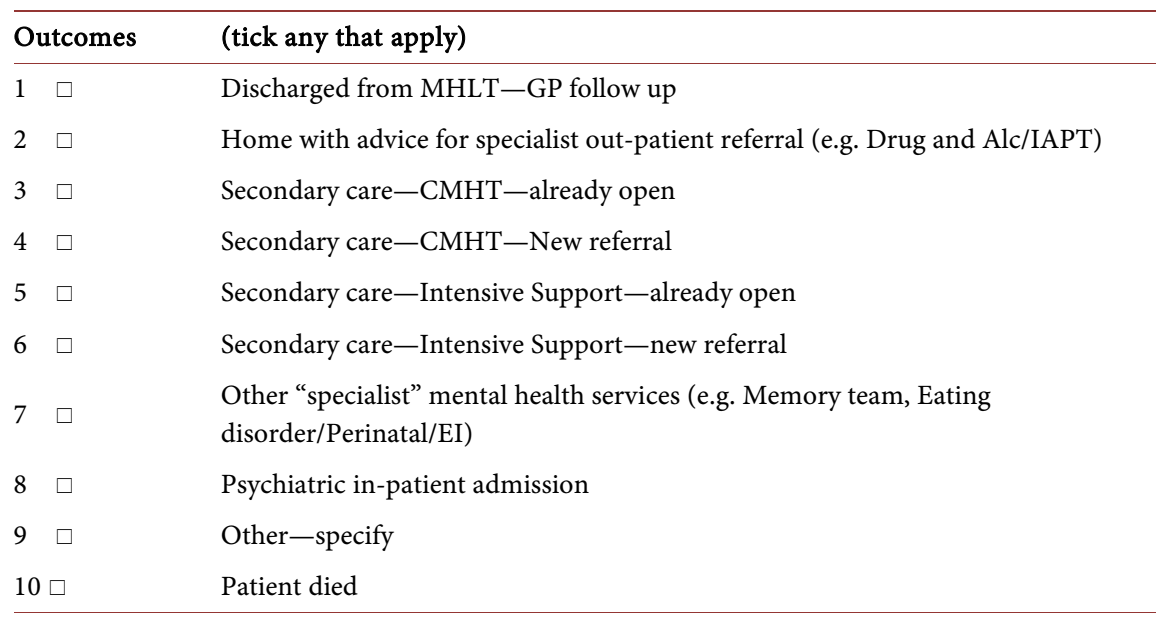

CGI-I: Clinical Global Impression-Improvement scale (not for one off contacts e.g. ED dept)

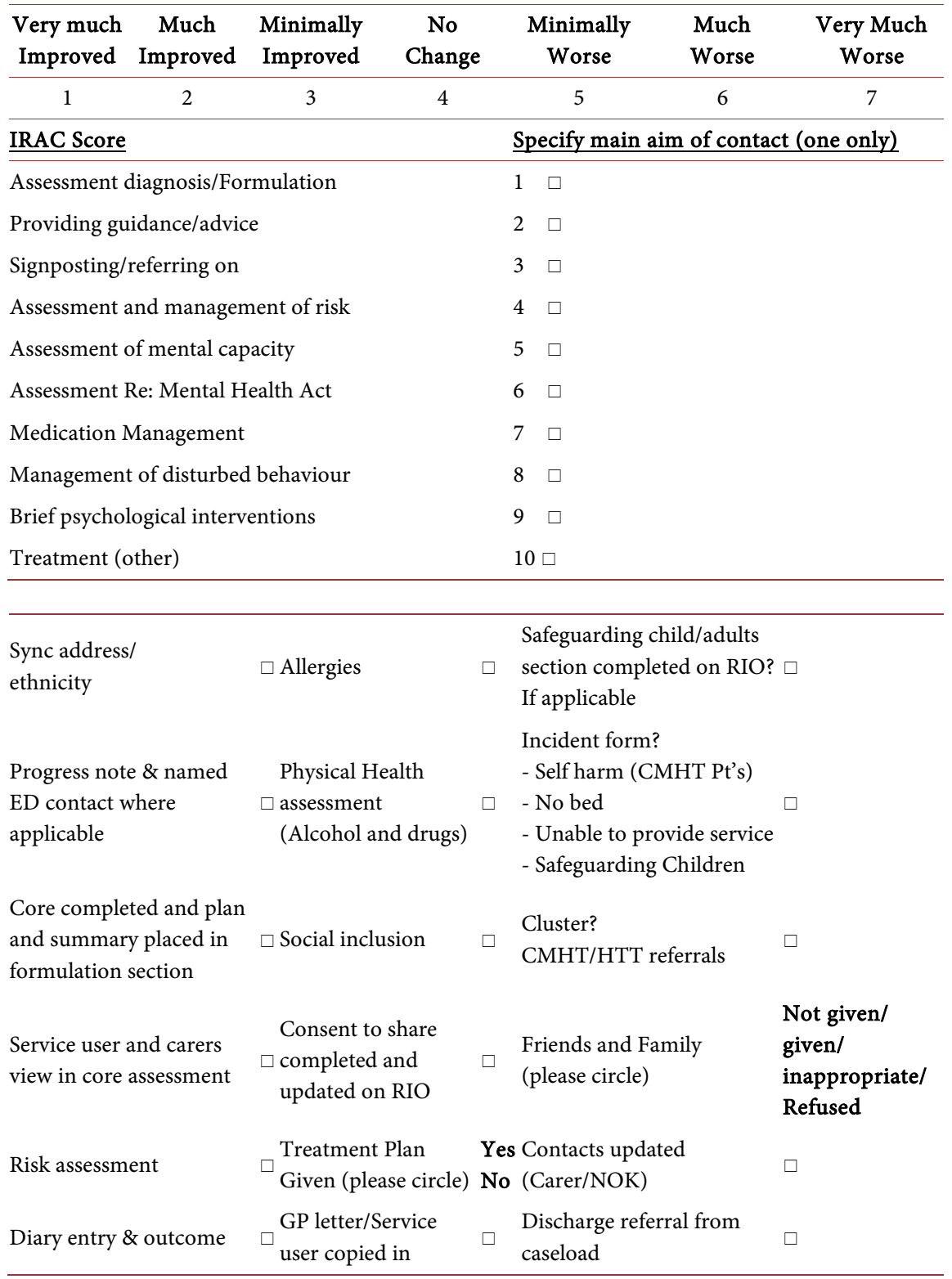

